\title{
Atuação da fisioterapia intensiva no contexto da pandemia de Covid-
}

\section{9}

A COVID-19, doença causada pelo novo coronavírus, está afetando consideravelmente as práticas de saúde da maioria dos países. Ela se apresenta com sintomas respiratórios, culminando algumas vezes, em morte ocasionada por Síndrome Respiratória Aguda Grave. Esse contexto exigiu uma mudança na organização dos sistemas de saúde, novos protocolos e alteração da atuação de profissionais, como do fisioterapeuta. A atuação desses profissionais no âmbito da reabilitação tem se mostrado muito útil ao prognóstico dos pacientes. Por isso, está busca revisar a literatura existente acerca da fisioterapia e reabilitação respiratória em pacientes portadores do novo coronavírus (SARS-CoV-2) e assim, levantar o atual estado dessa prática. Tratase de um trabalho qualitativo, básico, exploratório e bibliográfico integrativo. A busca foi realizada utilizando os descritores: Physical Therapy, Intesive Physiotherapy, Coronavirus Infection e Respiration, nos repositórios Pubmed, Bireme e Springerlink e foram incluídos trabalhos em inglês e francês de 2020 e 2021 . Os dados relatados sugerem que a fisioterapia fornece suporte e Respiration, nos repositórios Pubmed, Bireme e Springerlink e foram incluídos trabalhos em inglês e francês de 2020 e 2021 . Os dados relatados sugerem que a fisioterapia fornece suporte ventilatório durante a fase aguda da doença e intervenções de reabilitação posteriores a fim de promover a funcionalidade. Apesar de não existir evidências que ela altere o curso do COVID19 na fase aguda da doença em pacientes com insuficiência respiratória hipoxêmica e tosse seca, pacientes com tosse produtiva podem se beneficiar de manobras de higiene brônquica que estimulem a tosse. Fornecimento de oxigênio, ventilação mecânica não invasiva, desmame da ventilação assistida e desobstrução das vias aéreas são aplicados, manobras de mobilização principalmente um pal de monitoramento e posicionamento do paciente para otimizar a razão ventilação/perfusão. A posição prona é recomendada para pacientes em situação grave. Além disso, são garantidas todas as aç̃es que promovam a autonomia respiratória do paciente, quando as condicões clínicas permitem. Considera-se que há pouca base de dados relatados na litera posicionamento de pacientes com insuficiência respiratória grave. Evidentemente, o acompanhamento, no decorrer da enfermidade e na recuperação, é importante para o acometido que apresenta melhor evolução clínica.

Palavras-chave: Infecções por Coronavírus; Fisioterapia; Unidade de Terapia Intensiva; Serviço Hospitalar de Fisioterapia; Respiração.

\section{Intensive physiotherapy performance in the context of the pandemic of covid-19}

COVID-19, disease caused by the new coronavirus, is significantly affecting the health practices of most countries. She presents with respiratory symptoms, sometimes culminating in death caused by Severe Acute Respiratory Syndrome. This context required a change in the organization of health systems, new protocols and changes in the performance of professionals, such as physiotherapists. The performance of these professionals in the context of rehabilitation has proved to be very useful in the prognosis of patients. For this reason, it seeks to review the existing literature on physical therapy and respiratory rehabilitation in patients with the new coronavirus (SARS-CoV-2) and, thus, to raise the current state of this practice. It is a qualitative, basic, exploratory and bibliographic integrative work. The search was performed using the descriptors: Physical Therapy, Intesive Physiotherapy, Coronavirus Infection and Respiration, in the Pubmed, Bireme and Springerlink repositories, and works in English and French from 2020 and 2021 were included. The reported data suggest that physiotherapy provides ventilatory support during the acute phase of the disease and subsequent rehabilitation interventions to promote functionality. Although there is no evidence that it changes the course of COVID-19 in the acute supply, noninvasive mechanical ventilation, weaning from assisted ventilation and airway clearance are applied, early mobilization maneuvers and posture variation are performed daily. However, for patients with invasive mechanical ventilation, there are few indications of physical therapy work, which mainly has a role in monitoring and positioning the patient to optimize the ventilation/perfusion ratio. The prone position is recommended for severely ill patients. In addition, all actions that promote the patient's respiratory autonomy are guaranteed, when clinical conditions permit. It is considered that there is little database reported in the literature for a concise discussion about the proposed theme. However, it can be inferred that there is evidence that physical therapy for patients affected by COVID-19 helps with prognosis, as it provides better oxygenation and gas exchange, in addition to offering airway clearance techniques for ventilated and auxiliary patients. in the positioning of patients with severe respiratory failure. Evidently, monitoring, during the course of the disease and in recovery, is important for the patient with the best clinical evolution.

Keywords: Coronavirus Infections; Physical Therapy; Intensive Care Units; Physical Therapy Department; Respiration.

Topic: Fisioterapia

Reviewed anonymously in the process of blind peer.

Álvaro José Ramos Feijó Silva (iD

Centro Universitário Brasileiro, Brasil

http://lattes.cnpq.br/8310523695918417

http://orcid.org/0000-0003-0167-2303

alvaro.ramos286@gmail.com

Marcos Lorran Paranhos Leão (ic

Universidade de Pernambuco, Brasil

http://lattes.cnpq.br/7449167140800038

http://orcid.org/0000-0002-6259-2430

upeleao@gmail.com

Suellen de Oliveira Paranhos (iD)

Universidade de Pernambuco, Brasil

http://lattes.cnpq.br/3900709889572098

http://orcid.org/0000-0002-1013-9415

su fisio@yahoo.com.br

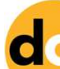

DOI: 10.6008/CBPC2674-6506.2021.001.0001
Received: $\mathbf{2 0 / 1 0 / 2 0 2 0}$

Approved: 08/02/2021
Referencing this:

SILVA, Á, J. R. F.; LEÃO, M. L. P.; PARANHOS, S. O.. Atuação da fisioterapia intensiva no contexto da pandemia de covid-19. Health of Humans, v.3, n.1, p.1-7, 2021. DOI: http://doi.org/10.6008/CBPC2674$\underline{6506.2021 .001 .0001}$ 


\section{INTRODUÇÃO}

O surto da doença coronavírus 2019 (COVID-19) se iniciou na Província de Hubei, na República Popular da China (WESTON et al., 2020; WHO, 2020), e em 11 de março de 2020, o Comitê de Emergência da Organização Mundial de Saúde declarou uma pandemia (WHO, 2020). Hoje, 21 de janeiro de 2021, já atingiu mais de 111 milhões e 277 mil pessoas, além de causar mais de 2 milhões 464 mil mortes ao redor do mundo.

Diversos sintomas podem se manifestar em infectados pelo vírus, mas os mais comuns incluem febre (89\%), tosse (68\%), fadiga (38\%), produção de escarro (34\%) e falta de ar (19\%) (GUAN, 2020). Uma parte considerável da população com COVID-19 pode não necessitar de internação, pois a maioria dos pacientes apresentam uma forma leve da doença com prognóstico favorável (YANG et al., 2020). No entanto, pacientes mais velhos e portadores de doenças crônicas, que se encontram no atual grupo de risco, podem apresentar complicações como Síndrome de Sofrimento Respiratório Agudo (ARDS), sepse, choque séptico, insuficiência renal e cardíaca, e até desenvolver doenças graves que necessitam de tratamento em Unidade de Terapia Intensiva (UTI) com apoio invasivo (YANG et al., 2020).

Em diversos países, os fisioterapeutas têm reconhecimento como profissionais que atuam na atenção primária e terciária (JERRE et al., 2007; STILLER, 2013; SPRUIT, 2014) com um papel fundamental em equipes multiprofissionais, promovendo intervenções de reabilitação posteriores para uma promoção da funcionalidade e também suporte ventilatório durante a fase de adoecimento agudo (AMBROSINO et al., 2013; GOÃI-VIGURIA et al., 2018).

Os pacientes incorrem em sarcopenia, fraqueza e diminuição da tolerância ao exercício devido ao longo período de repouso em isolamento ou tratamento na UTI necessitando do profissional fisioterapeuta no auxílio do caso (ZHU et al., 2020). Atualmente entre os fisioterapeutas o método para reabilitar pacientes com segurança COVID-19 é uma questão que tem levado a preocupações (ZHU et al., 2020).

A permanência prolongada em cuidados intensivos acarreta consequências sobretudo respiratórias e ortopédicas. Mas, além dos pacientes que precisam de uma passagem na terapia intensiva, o quadro clínico relacionado a COVID-19 leva outros pacientes a necessitarem de reabilitação (Prise en charge précoce de médecine physique et de réadaptation (MPR) en réanimation en soins continus ou en service de rééducation post-réanimation (SRPR), citado por SMONDACK et al., 2020). Portanto, o cuidado fisioterapêutico é baseado em 3 aspectos: o aspecto respiratório, o aspecto motor e a prevenção de complicações (SMONDACK et al., 2020).

No entanto, sintomas respiratórios pré-existentes como tosse seca e a produção de escarro podem progredir por algum tempo durante o período de reabilitação, o que pode ocasionar a diminuição da qualidade de vida (ZHA et al., 2020). Para os grupos de risco, principalmente idosos e pacientes com distúrbios respiratórios pré-existentes (por exemplo, DPOC, doenças pulmonares restritivas), é difícil esperar aliviar suavemente devido aos músculos respiratórios enfraquecidos (ZHA et al., 2020). No momento atual, vários exercícios são introduzidos para ajudar a enfrentar esses problemas durante o processo de recuperação da doença (KLIJN et al., 2013; GREENING et al., 2014; CECINS, 2017; VAN DER LEE et al., 2018; 
LÓPEZ-LÓPEZ et al., 2019). Dessa forma, a maioria deles se concentra apenas no treinamento de músculos respiratórios, sem a devida atenção às alterações patológicas específicas do COVID-19 (ZHA et al., 2020).

A atuação desses profissionais no âmbito da reabilitação tem se mostrado muito útil ao prognóstico dos pacientes (SAEKI et al., 2020). Portanto, devido a atual necessidade dessa arte, a urgência em se obter mais conhecimentos na área e a complexidade da questão, este trabalho objetiva revisar a literatura existente, que atenda os critérios de inclusão, acerca da fisioterapia intensiva em pacientes portadores do novo coronavírus (SARS-CoV-2) e assim, levantar o atual estado dessa prática.

\section{METODOLOGIA}

O presente estudo é um trabalho qualitativo, básico, exploratório e bibliográfico. Trata-se de uma revisão integrativa ( $n=23$ ) em que a busca foi realizada utilizando-se os Descritores em Ciências da Saúde (DeCS): Physical Therapy, Intesive Physiotherapy, Coronavirus Infection and Respiration, nos repositórios Pubmed, Bireme e Springerlink. Foram incluídos trabalhos em inglês e francês de 2020 e 2021, além de literatura histórica anterior. Além disso, por esta ser uma pesquisa que utiliza informações de acesso público, Lei $n^{\circ} 12.527$ (18 de novembro de 2011), não precisa de autorização em comitê de ética [parágrafo único do Art $1^{\circ}$ da resolução 510/2016 da Comissão Nacional de Ética em Pesquisa (CONEP) e do Conselho Nacional de Saúde (CNS) (CNS et al., 2016)].

\section{DISCUSSÃO TEÓRICA}

Após a análise dos títulos selecionados, se pode inferir que poucas literaturas estão disponíveis sobre a fisioterapia durante a pandemia COVID-19 (LAZZERI et al., 2020). Contudo, a fisioterapia já se mostrou eficaz para melhorar a função física a longo prazo entre os pacientes que deixam a Unidade de Tratamento Intensivo (UTI) (CALVO-AYALA et al., 2013). Da mesma maneira, a mobilização e reabilitação precoces podem ajudar a prevenir e/ou mitigar sequelas relacionadas a grande estadia no leito, reduzindo o tempo de permanência e auxiliando na ventilação mecânica (KAYAMBU et al., 2013).

No contexto COVID-19, foi descrito que a fisioterapia respiratória fornece suporte ventilatório durante a fase aguda da doença e também intervenções de reabilitação posteriores que podem promover a melhor funcionalidade pulmonar (RIGHETTI et al., 2020). A fisioterapia iniciada de forma precoce, ou seja, durante a ventilação mecânica, é considerada viável e segura para que o desempenho e a qualidade de vida do paciente melhore (KAYAMBU et al., 2013), ainda que isso não tenha sido comprovado no COVID-19 (BATTAGLINI et al., 2020) na fase aguda da doença em pacientes com tosse seca e insuficiência respiratória hipoxêmica (RIGHETTI et al., 2020), as manobras de higiene brônquica que estimulam a tosse podem beneficiar os pacientes com tosse produtiva (ZHA et al., 2020).

Sendo assim, a fisioterapia pode ser indicada para pacientes com COVID-19 que apresentam grande volume de secreções nas vias aéreas e não são capazes de limpar de forma independente (ANZICS, 2020). Consequentemente, os fisioterapeutas que atuam em ambiente de UTI também podem oferecer técnicas de desobstrução das vias aéreas para pacientes ventilados com a presença de sinais de desobstrução 
inadequada das vias aéreas e auxiliando no posicionamento de pacientes com insuficiência respiratória grave associada ao COVID-19, incluindo o uso da posição prona para otimizar a oxigenação (ANZICS, 2020). Entre as estratégias de fisioterapia respiratória frequentemente aplicadas na prática clínica nesse período , cerca de 34\% dos pacientes com COVID-19 teve uma produção de escarro relatada (BATTAGLINI et al., 2020; GUAN et al., 2020), sugerindo que, ao promover o desembaraço do muco durante a ventilação mecânica, intervenções de fisioterapia precoce (como drenagem de secreção subglótica, higiene postural e hiperinflação do ventilador) efeitos benéficos podem ser produzidos nesta nova população gravemente doente (THOMAS et al., 2020).

A oxigenoterapia representa uma intervenção importante para pacientes com disfunção pulmonar grave e a instituição de ventilação mecânica pode ser necessária em insuficiência respiratória refratária à oxigenoterapia (RIGHETTI et al., 2020). Fornecimento de oxigênio, ventilação mecânica não invasiva, desobstrução das vias aéreas e desmame da ventilação assistida são aplicados, além de que a mobilização precoce e a variação da postura são efetuadas cotidianamente (SMONDACK et al., 2020). Contudo, para pacientes com ventilação mecânica invasiva, há poucas indicações de trabalho fisioterápico, que exercem principalmente um papel de monitoramento, particularmente o posicionamento do paciente para otimizar a razão ventilação/perfusão (SMONDACK et al., 2020). O decúbito ventral é recomendado de 12 a 16 horas por dia para pacientes com ER grave devido aos seus efeitos benéficos sobre a mortalidade (HU et al., 2014) para pacientes com SRAG grave é recomendada a posição prona (RIGHETTI et al., 2020).

Além disso, são asseguradas todas as ações que promovam a autonomia respiratória do paciente, quando as condições clínicas permitem (LAZZERI, 2020). Podem ser avaliados caso a caso, e as intervenções são aplicadas baseadas em indicadores clínicos (ANZICS, 2020). A fisioterapia para pacientes críticos em geral, em doenças críticas e pós-críticas, é apoiada em uma abordagem multissistêmica que compreende não só a fisioterapia respiratória, mas também a reabilitação musculoesquelética, a fim de reduzir a incidência de complicações, incentivar o desmame da ventilação mecânica e favorecer recuperação da autonomia funcional (THOMAS et al., 2020).

O verdadeiro benefício da fisioterapia respiratória na UTI permanece controverso, especialmente naqueles pacientes com danos alveolares já estabelecidas (LAZZERI et al., 2020; THOMAS et al., 2020). A Associação Italiana de Fisioterapeutas Respiratórios (ARIR) publicou recentemente um artigo de posição sobre o papel da fisioterapia respiratória em pacientes com COVID-19 (LAZZERI et al., 2020), sugerindo a limitação de alguns procedimentos, como respiração diafragmática, higiene brônquica, técnicas de reexpansão pulmonar, mobilização manual, treinamento muscular respiratório, lavagem nasal e treinamento de exercícios, na fase aguda da doença. A literatura sugere que as manobras da fisioterapia resultam em mudanças significativas na função respiratória (CERQUEIRA NETO et al., 2013), bem como em alterações hemodinâmicas cardiovasculares e cerebrais (CERQUEIRA NETO et al., 2013), o que poderia levar a efeitos potencialmente prejudiciais (BATTAGLINI et al., 2020).

A fisioterapia precoce do tórax pode ser considerada em todos os pacientes COVID-19 que necessitam de ventilação mecânica, durante e após o processo de extubação (BATTAGLINI et al., 2020). As 
técnicas mais frequentes aplicadas após a extubação incluem estimulação elétrica neuromuscular, sessão inicial, sucção das vias aéreas, hiperinflação manual, técnicas de limpeza das vias aéreas, triagem de andorinhas, mobilização precoce, pressão expiratória positiva com dispositivo EzPAP, pressão expiratória positiva, ciclo ativo de técnicas de respiração (ACBT), respiração positiva intermitente, técnica expiratória forçada, manobras de tosse assistidas ou estimuladas, insuficiência-sufocamento, CPAP, NIV e HFNO (LAZZERI et al., 2020).

$\mathrm{Na}$ fisioterapia respiratória, a drenagem brônquica não é indicada em casos de sintomas moderados sem impacto respiratório significativo (sem febre, tosse produtiva ou alterações no raio-x do tórax, etc.) ou em casos de doença pulmonar com requisitos de oxigênio baixos, tosse não produtiva ou quando o paciente é capaz de drenar sozinho (THOMAS et al., 2020). A drenagem brônquica, no entanto, é indicada em pacientes com doença pulmonar ou baixa infecção respiratória com tosse ineficaz e produtiva e com complicações na declinação brônquica, com ou sem histórico de patologias respiratórias ou neuromusculares. Em caso de intervenção na esfera respiratória, o paciente, se possível, deve usar uma máscara cirúrgica (a ser trocada a cada 4 horas) (THOMAS et al., 2020) (Avis relatif aux conditions de prolongation du port ou de réutilisation des masques chirurgicaux et des appareils de protection respiratoire de type FFP2 pour les professionnels de santé, citado por SMONDACK et al., 2020).

Além desse período, a reabilitação respiratória pode ser prevista, aplicando os princípios usuais, adaptados ao paciente (SMONDACK et al., 2020). O programa de reabilitação será adaptado para cada paciente com base nas suas possibilidades funcionais e sua pré-avaliação. Como o paciente é avaliado nas condições habituais, os princípios de reassunção ao estresse são os mesmos do contexto de outra patologia (SMONDACK et al., 2020). No entanto, devem ser tomadas precauções devido à pandemia. O acompanhamento clínico deve ser adaptado caso a caso de acordo com as comorbidades do paciente (SPRUIT et al., 2013).

A predominância de tosse seca, tosse produtiva, dificuldade de expectativa e dispneia foi de $41,7 \%$, $43,3 \%, 35,0 \%$ e 50,0\%, respectivamente, que reduziram acentuadamente ao longo do tempo de atendimento fisioterápico (ZHA et al., 2020). Após um mês, a predominância foi de $11,7 \%$ na tosse seca, $11,7 \%$ na tosse produtiva, $8,3 \%$ em dificuldade de expectativa e apenas $15 \%$ dos pacientes relataram dispneia como sintoma remanescente (ZHA et al., 2020). De acordo com a taxa de remissão retratada dessas manifestações clínicas em quatro pontos de tempo diferentes, pacientes com casos leves que executaram técnicas de treinamento dos exercícios de reabilitação modificados (MRE) apresentaram maior taxa de remissão em relação aos valores da linha de base (ZHA et al., 2020). Vale ressaltar que os sintomas refratários ainda perduram em alguns pacientes, o que colaborou para uma menor taxa de remissão na tosse produtiva e dificuldade na expectativa após quatro semanas de alta (ZHA et al., 2020).

\section{CONCLUSÕES}

Considera-se que há pouca base de dados relatados na literatura para uma discussão concisa acerca do tema proposto. Contudo, pode-se inferir que existem evidências de que a fisioterapia para o paciente 
acometido pela COVID-19 auxilia no prognóstico, na medida em que proporciona melhor oxigenação e trocas gasosas, além de oferecer técnicas de desobstrução das vias aéreas para pacientes ventilados auxiliar no posicionamento de pacientes com insuficiência respiratória grave. Ainda, reconhece-se que a fisioterapia nesse contexto atua não somente na área respiratória, mas também com a reabilitação musculoesquelética, a fim de reduzir a incidência de complicações, estimular o desmame da ventilação mecânica e facilitar a recuperação da autonomia funcional. Evidentemente, o acompanhamento, no decorrer da enfermidade e na recuperação, é importante para o acometido que apresenta melhor evolução clínica.

\section{REFERÊNCIAS}

AMBROSINO, N.; MAKHABAH, D. N.. Comprehensive physiotherapy management in ARDS. Minerva Anestesiol, v.79, n.5, p.554-563, 2013.

BATTAGLINI, D.; ROBBA, C.; CAIFFA, S.; BALL, L.; BRUNETTI, I.; LOCONTE, M.; GIACOBBE, D. R.; VENO, A.; PATRONI, N.; BASSETI, M.; TORRES, A.; ROCCO, P. R.; PELOSI, P.. Chest physiotherapy: An important adjuvant in critically ill mechanically ventilated patients with COVID-19. Respir Physiol Neurobiol, v.282, p.103529, 2020. DOI: http://doi.org/10.1016/j.resp.2020.103529

CALVO-AYALA, E.; KHAN, B. A.; FARBER, M. O.; WESLEY ELY, E.; BOUSTANI, M. A.. Intervenções para melhorar a função física dos sobreviventes da UTI: uma revisão sistemática. 0 peito, v.144, p.1469-1480, 2013.

CECINS, N.; LANDERS, H.; JENKINS, S.. A reabilitação pulmonar baseada na comunidade em uma instalação nãomédica é viável e eficaz. Chron Respir Dis, v.14, p.3-10, 2017.

CERQUEIRA NETO, M.; MOURA, A.; CERQUEIRA, T.; AQUIM, E.; REA NETO A.; OLIVEIRA, M.; SILVA JUNIOR, W.; SANTANA FILHO, V.; SCOLA, R.. Efeitos agudos de manobras respiratórias fisioterapêuticas em pacientes gravemente doentes com trauma craniocerebral. Clínicas, v.68, p.12101214, 2013.

CNS; MES. Comitê de Ética em Pesquisa; Comissão Nacional de Ética em Pesquisa. Resolução № 510, de 07 de abril de 2016. Brasília: CNS, 2016.

GOÃI-VIGURIA, R.; VOLDI-ARVOZ, E.; CASAJÚS-SOLA, L.; AQUERRETA-LARRAYA, T.; FERNÁNDEZ-SANGIL, P.; GUZMÁN-UNAMUNO, E.; MOYANO-BERARDO, B. M.. Respiratory Physiotherapy in Intensive Care Unit: Bibliographic Review. Enferm Intensiva, v.29, n.4, p.168-181, 2018. DOI: http://doi.org/10.1016/j.enfi.2018.03.003

GREENING, N. J.; WILLIAMS, J. E. A.; HUSSAIN, S. F.; HARVEYDUNSTAN, T. C.; BANKART, M. J.; CHAPLIN, E. J.; VINCENT, E. E.; CHIMERA, R.; MORGAN, M. D.; SINGH, S. J.; STEINER, M. C.. Uma intervenção de reabilitação precoce para melhorar a recuperação durante a internação hospitalar para uma exacerbação de doença respiratória crônica: ensaio controlado randomizado. BMJ, v.349, n.4315, 2014.

GUAN, W. J.; NI, Z. Y.; HU, Y.; LIANG, W. H.; OU, C. Q.; HE, J. X.; LIU, L.; SHAN, H.; LEI, C. L.; HUI, D. S. C.; DU, B.; LI, L. J.; ZENG, G.; YUEN, K. Y.; CHEN, R. C.; TANG, C. L.; WANG, T.;
CHEN, P. Y.; XIANG, J.; LI, S. Y.; WANG, J. L.; LIANG, Z. J.; PENG, Y. X.; WEI, L.; LIU, Y.; HU, Y. H.; PENG, P.; WANG, J. M.; LIU, J. Y.; CHEN, Z.; LI, G.; ZHENG, Z. J.; QIU, S. Q.; LUO, J.; YE, C. J.; ZHU, S. Y.; ZHONG, N. S.. China Medical Treatment Expert Group for Covid-19. Clinical Characteristics of Coronavirus Disease 2019 in China. N. Engl. J. Med., v.382, n.18, p.1708-1720. DOI: http://doi.org/10.1056/NEJMoa2002032

HU, S. L.; HE, H. L.; PAN, C.. The effect of prone positioning on mortality in patients with acute respiratory distress syndrome: a meta-analysis of randomized controlled trials. Crit Care, v.18, n.3, p.109, 2014.

JERRE, G.; SILVA, T. J.; BERALDO, M. A.; GASTALDI, A.; KONDO, C.; LEME, F.; GUIMARÃES, F.; FORTI JUNIOR, G.; LUCATO, J. J.; TUCCI, M. R.; VEGA, J. M.; OKAMOTO, V. N.. Physiotherapy on the Mechanically Ventilated Patients. J. Bras Pneumol, v.33, n.2, p.142-50, 2007. DOI: http://doi.org/10.1590/S1806-37132007000800010

KAYAMBU, G.; BOOTS, R.; PARATZ, J.. Fisioterapia para doentes graves na UTI: revisão sistemática e meta-análise. Cuidado Med, v.41, p.1543-1554, 2013.

KLIJN, P.; VAN KEIMPEMA, A.; LEGEMAAT, M.; GOSSELINK, R.; VAN STEL, H.. Nonlinear Exercise Training in Advanced Chronic Obstruive Pulmonary Disease Is Superior to Traditional Exercise Training. Um julgamento randomizado. Am J. Respir Crit Care Med, v.188, p.193-200, 2013.

LAZZERI, M.; LANZA, A.; BELLINI, R.; BELLOFIORE, A.; CECCHETTO, S.; COLOMBO, A.; D'ABROSCA, F.; DEL MONACO, C.; GAUDELLIO, G.; PANERONI, M.; PRIVITERA, E.; RETUCCI, M.; ROSSI, V.; SANTAMBROGIO, M.; SOMMARIVA, M.; FRIGERIO, P.. Respiratory physiotherapy in patients with COVID-19 infection in acute setting: a Position Paper of the Italian Association of Respiratory Physiotherapists (ARIR). Monaldi Arch, v.90, 2020. DOI: http://doi.org/10.4081/monaldi.2020.1285

LAZZERI, M.. How Italian respiratory physiotherapists have faced and are facing the coronavirus disease 2019 pandemic. Arch Physiother, v.15, 2020.

LÓPEZ-LÓPEZ, L.; TORRES-SÁNCHEZ, I.; RODRÍGUEZ-TORRES, J.; CABRERA-MARTOS, I.; ORTIZ-RUBIO, A.; VALENZA, M. C.. Does adding an integrated physical therapy and neuromuscular electrical stimulation therapy to standard rehabilitation improve functional outcome in elderly patients with pneumonia? A randomised controlled trial. 
Clin Rehabil, v.33, n.11, p.1757-1766, 2019. DOI: http://doi.org/10.1177/0269215519859930

RIGHETTI, R. F.; ONOUE, M. A.; POLITI, F. V.A.; TEIXEIRA, D. T.; SOUZA, P. N.; KONDO, C. S.; MODERNO, E. V.; MORAES, I. G.; MAIDA, A. L. V.; PASTORE JUNIOR, L.; SILVA, F. D.; BRITO, C. M. M.; BAIA, W. R. M.; YAMAGUTI, W. P.. Physiotherapy Care of Patients with Coronavirus Disease 2019 (COVID-19) A Brazilian Experience. Clinics, v.75, e.2017, 2020. DOI: https://dx.doi.org/10.6061/clinics/2020/e2017

SAEKI, T.; OGAWA, F.; CHIBA, R.; NONOGAKI, M.; UESUGI, J.; TAKEUCHI, I.; NAKAMURA, T.. Rehabilitation Therapy for a COVID-19 Patient Who Received Mechanical Ventilation in Japan. Am J. Phys Med Rehabil, v.99, n.10, p.873-875, 2020. DOI: http://doi.org/10.1097/PHM.0000000000001545

SMONDACK ET AL.,P.; GRAVIER, F. É.; PRIEUR, G.; REPEL, A.; MUIR, J. F.; CUVELIER, A.; COMBRET, Y.; MECRINAL, C.; BONNEVIE, T.. Kinésithérapie et COVID-19: de la réanimation à la réhabilitation à domicile. Synthèse des recommandations internationales. Revue des maladies respiratoires, v.37, n.10, 811-822, 2020. DOI: https://doi.org/10.1016/j.rmr.2020.09.001

STILLER, K.. Physiotherapy in intensive care: an updated systematic review. Chest, v.144, n.3, p.825-47, 2013. DOI: http://doi.org/10.1378/chest.12-2930

SPRUIT, M. A.. Pulmonary rehabilitation. Eur Respir Rev, v.23, n.131, p.55-63, 2014. DOI: http://doi.org/10.1183/09059180.00008013

SPRUIT, M. A.; SINGH, S. J.; GARVEY, C.. An official American Thoracic Society/European Respiratory Society statement: key concepts and advances in pulmonary rehabilitation. Am J. Respir Crit Care Med, v.188, n.8, P.13-64, 2013.

THOMAS, P.; BALDWIN, C.; BISSETT, B.; BODEN, I.; GOSSELINK, R.; GRANGER, C. L.; HODGSON, C.; YM, A.;
MICHELLE, J.; KHO, E.; MOSES, R.; NTOUMEN, G.; PARRY, S. M.; PATMAN, S.; VAN DER LEE, L.. Physiotherapy management for COVID-19 in the acute hospital setting: clinical practice recommendations. Physiother, v.66, p.7382,2020

VAN DER LEE, L.; HILL, A. M.; PATMAN, S.. Fisioterapia respiratória após o expediente para pacientes entubados e mecanicamente ventilados com pneumonia adquirida pela comunidade: uma perspectiva australiana. Aust Crit Care, v.31, p.349-54, 2018.

WESTON, S.; FRIEMAN, M. B.. COVID-19: Knowns, Unknowns, and Questions. MSphere, v.5, n.2, p.20172020. DOI: http://doi.org/10.1128/mSphere.00203-20

WHO. World Health Organization. Clinical management of severe acute respiratory infection when COVID-19 is suspected. WHO, 2020.

YANG, X.; YU, Y.; XU, J.; SHU, H.; XIA, J.; LIU, H.; ZHANG, L.; YU, Z.; FANG, M.; YU, T.; WANG, Y.; PAN, S.; ZOU, X.; YUAN, S.; SHANG, Y.. Clinical Course and Outcomes of Critically III Patients With SARS-CoV-2 Pneumonia in Wuhan, China: A Single-Centered, Retrospective, Observational Study. Lancet Respir Med, v.8, n.5, p.475-481, 2020. DOI: http://doi.org/10.1016/S2213-2600(20)30079-5

ZHA, L.; XU, X.; WANG, D.; QIAO, G.; ZHUANG, W.; HUANG, S.. Modified rehabilitation exercises for mild cases of COVID19. Ann Palliat Med, v.9, n.5, p.3100-3106, 2020. DOI: http://doi.org/10.21037/apm-20-753

ZHU, Y.; WANG, Z.; ZHOU, Y.; ONODA, K.; MARUYAMA, H.; HU, C.; LIU, Z.. Summary of respiratory rehabilitation and physical therapy guidelines for patients with COVID-19 based on recommendations of World Confederation for Physical Therapy and National Association of Physical Therapy. J. Phys Ther Sci, v.32, n.8, p.545-549, 2020. DOI: http://doi.org/10.1589/jpts.32.545

A CBPC - Companhia Brasileira de Produção Científica (CNPJ: 11.221.422/0001-03) detém os direitos materiais desta publicação. Os direitos referem-se à publicação do trabalho em qualquer parte do mundo, incluindo os direitos às renovações, expansões e disseminações da contribuiç̃o, bem como outros direitos subsidiários. Todos os trabalhos publicados eletronicamente poderão posteriormente ser publicados em coletâneas impressas sob coordenação da Sapientiae Publishing, da Companhia Brasileira de Produção Científica e seus parceiros autorizados. Os (as) autores (as) preservam os direitos autorais, mas não têm permissão para a publicação da contribuição em outro meio, impresso ou digital, em português ou em tradução. 